TP Periodica Polytechnica

Transportation Engineering

47(1), pp. 64-67, 2019

https://doi.org/10.3311/PPtr. 11548

Creative Commons Attribution (i)

RESEARCH ARTICLE

\section{Static System Optimum of Linear Traffic Distribution Problem Assuming an Intelligent and Autonomous Transportation System}

\author{
Gábor Pauer ${ }^{1 *}$, Árpád Török ${ }^{1}$
}

Received 24 January 2017; accepted 03 October 2017

\begin{abstract}
The aim of this article is to present the research results of the authors in the field of defining the system optimum regarding the traffic distribution assuming an intelligent and autonomous transportation system. Authors define the linear programming framework of the traffic distribution problem in case of pre-defined demand structure, network properties and alternative routes related to each origin-destination zone pair. In the paper the description of the applied method and its verification are presented in a simplified model example. As a basic result, it is clearly shown that in case of the introduced static conditions the approach is applicable to determine the optimal solution of the traffic distribution problem in a given sample time period of an autonomous transportation system.
\end{abstract}

\section{Keywords \\ traffic distribution, linear programming, autonomous system, system optimization}

${ }^{1}$ Department of Transport Technology and Economics,

Faculty of Transportation Engineering and Vehicle Engineering,

Budapest University of Technology and Economics,

H-1521 Budapest, P.O.B. 91, Hungary

*Corresponding author, e-mail: pgabor90@gmail.com

\section{Introduction}

Traffic distribution problem has been the subject of many scientific researches. The basic methodological considerations are well discussed by the related literatures (Yang, 2016). Most of the researches focused on business oriented application possibilities especially considering the field of freight transportation and logistics. Díaz-Madroñero, Mula and Peidro have proposed a new mathematical programming model for integrating production and procurement transport planning decisions in manufacturing systems in a unique optimization model (DíazMadroñero et al., 2017) but they do not discuss the application possibilities of their approach in case of a passenger transportation system. Harasimowicz, Janus, Bacior and Gniadek present a new approach to the problem of optimization of land arrangement. The introduced optimization model takes into account a number of very important factors in the process of land consolidation, including map of the land diversification or the actual shape of the transportation network (Harasimowicz et al., 2017) however the implemented methodology is rather applicable to handle long-term problems than support such short-term problems like real time traffic distribution in urban environment. Ma, Li, Zhou, and Hao investigate a new vehicle sharing system using autonomous vehicles. In such a system, travelers can request autonomous vehicle trips ahead of time and the system operator will optimally arrange autonomous vehicles pickup and delivery schedules and autonomous vehicles trip chains based on these requests (Ma et al., 2017). Their system handles the problem from a vehicle fleet operation point of view and do not emphasize the question of constrained infrastructure capacity. Piyush Kumar, Jay Rosenbergery, and Gazi Iqbalz approach the problem from the aspect of operating urban environment and in their research; they develop a mixed integer programming model to optimize land usage subject to sprawl constraints, which are based upon sprawl measures (Kumar et al., 2016). but their method focuses on to solve long-term problems instead of short-term problems like traffic distribution of the urban system. Yakimov presents methodology of construction of mathematical model enabling proper distribution of transport demand within the city territory but his model also 
tries to find answers to the questions of overall urban planning approach and do not emphasize traffic engineering considerations (Yakimov, 2017).

\section{Methodology}

The aim of our research was to define the minimum of total travel time depending on total traffic appeared on a transportation network in a sample time period of the model. The investigated transport system and its components were assumed to be fully autonomous. This enables us to order trip distribution tasks and route choice decision processes under the control of the system, providing the necessary requirements to achieve system optimum. Model variables were the traffic volumes appearing on the alternative routes connecting origin-destination (henceforth: OD) zones, referred in the study as part-flow; the minimized objective function has been derived from the sum of products of part-flows and related travel times.

In our example, as a verification of the elaborated method, the transport network was constructed according to the graph indicated in Fig. 1, where the possible origin zones were $\mathrm{S}$ and $\mathrm{T}$ and the possible destination zones were $\mathrm{W}$ and $\mathrm{Z}$.

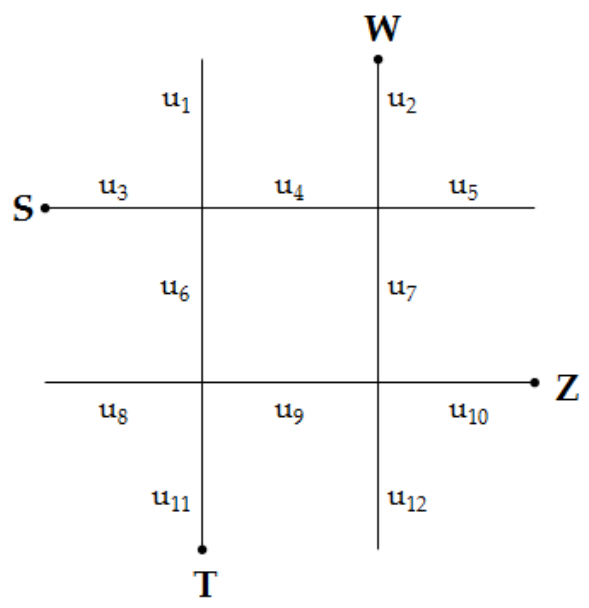

Fig. 1 Graph representing the investigated transportation network

There exist several alternative routes to reach a selected destination zone from an arbitrary origin zone. Routes are built up from graph edges $\left(u_{j}\right)$. Every edge can be characterized with constant capacity; where $c_{j}$ can be defined as the maximum number of users, who can use the investigated network component in a given sample time period and with constant travel time, where $t_{j}$ can be defined as the time needed to travel through the investigated network element. To determine a static system optimum of the autonomous transportation system, these characteristics of the edges $\left(c_{j}, t_{j}\right)$ as well as transport demands have to be considered as constant, defined parameters. Transport demands of the system have been indicated in our study as $D_{S W}$ (transport demands from $\mathrm{S}$ zone to $\mathrm{W}$ zone), $D_{S Z}, D_{T W}$ and $D_{T Z}$.

The aim of the optimization was to supply travel demands by the minimum level of total travel time function.
The introduced example can be described as a linear programming problem. The variables are part-flows, the traffic volumes appearing on the alternative routes connecting origin destination zones, constrains are capacity values of the edges and the travel demands appeared in a sample time period between OD pairs. The minimized objective function is the sum of products of the part-flow and travel time values of edges in a sample time period.

In the first step, possible routes $\left(U_{i}\right)$ between the investigated OD zones have been defined as follows:

From $\mathrm{S}$ to $\mathrm{W}$ :

$U_{S W 1}: u_{3}-u_{4}-u_{2}$

$U_{S W 2}: u_{3}-u_{6}-u_{9}-u_{7}-u_{2}$

From $\mathrm{S}$ to $\mathrm{Z}$ :

$U_{S Z 1}: u_{3}-u_{4}-u_{7}-u_{10}$

$U_{S Z 2}: u_{3}-u_{6}-u_{9}-u_{10}$

From $\mathrm{T}$ to $\mathrm{W}$ :

$U_{T W 1}: u_{11}-u_{6}-u_{4}-u_{2}$

$U_{T W 2}: u_{11}-u_{9}-u_{7}-u_{2}$

From $\mathrm{T}$ to $\mathrm{Z}$ :

$U_{T Z 1}: u_{11}-u_{9}-u_{10}$

$U_{T Z 2}: u_{11}-u_{6}-u_{4}-u_{7}-u_{10}$

Travel demands have been distributed among the above introduced possible routes. Possible part-flows $\left(x_{i}\right)$ ordered to routes between the OD pairs have been presented below:

Part-flow of route $U_{S W 1}: x_{1}$

Part-flow of route $U_{S W_{2}}: x_{2}$

Part-flow of route $U_{S Z 1}: x_{3}$

Part-flow of route $U_{S Z 2}: x_{4}$

Part-flow of route $U_{T W 1}: x_{5}$

Part-flow of route $U_{T W 2}: x_{6}$

Part-flow of route $U_{T Z 1}: x_{7}$

Part-flow of route $U_{T Z 2}: x_{8}$

Since travel demands between OD zones have been considered as constants, it was possible to define the below presented boundary conditions related to the traffic volume of the routes (Eq. (1)-(4)) as follows:

$$
\begin{aligned}
& x_{1}+x_{2}=D_{S W} \\
& x_{3}+x_{4}=D_{S Z} \\
& x_{5}+x_{6}=D_{T W} \\
& x_{7}+x_{8}=D_{T Z}
\end{aligned}
$$

With other words, travel demands were known between the OD pairs, which have been distributed among the possible routes as part-flows, so the sum of part-flow values $\left(x_{i}\right)$ on the possible routes of an OD pair is equal to the travel demand between the investigated OD zones. Furthermore the value of $x_{i}$ was required to be a non-negative integer (as it was representing traffic volumes in the example), as defined in Eq. (5):

$$
x_{i} \geq 0 \text { and } x_{i} \text { is integer, } i=1 \ldots 8
$$


In the next step constraining inequalities derived from the given capacity constraints have been defined. Since the capacity constraints of all 12 edges were pre-defined, 12 inequalities applying the 8 variables had to be considered. Since $x_{i}$ values (optimized variables) describe traffic volume on a route among an OD pair, while constrains represent maximum capacity of edges of the network graph, it was necessary to express traffic volumes $\left(X_{u j}\right)$ appearing on the edges of the graph in terms of the optimized variables. To achieve this goal $\underline{\underline{A}}$ coefficient matrix had to be constructed to define the relationship of the edges and the possible routes. $\underline{\underline{A}}$ matrix has 8 columns and 12 lines and contains 0 and 1 values. An arbitrary line of the matrix determines which routes contain the given edge (focusing on a line of the matrix: the value of a matrix component is 0 if the given edge is not part of the route described by the investigated column) and in accordance with this an arbitrary column of the matrix determines which edges are part of the given route (focusing on a column of the matrix: the value of a matrix component is 0 if the given route does not contain the edge described by the investigated line). Constraining inequalities (Eq. (6)) have been derived from the above introduced constraints as follows:

Related to the $j$-th edge:

$$
X_{u_{j}}=\sum_{i=1}^{8} x_{i} * a_{j i} \leq c_{j} \quad, j=1 \ldots 12
$$

The left side of the inequalities defines the traffic volumes appearing on the given edge. The components of $\underline{\underline{A}}$ matrix $\left(a_{j i}\right.$ coefficients) represents the relationship of edges and routes. For example, edge $u_{2}$ was part of $U_{S W 1} ; U_{S W 2} ; U_{T W 1}$ and $U_{T W 2}$ routes and the traffic volumes of these routes have been defined as $x_{1} ; x_{2} ; x_{5}$ and $x_{6}$. In accordance with this, in the inequality describing the capacity of $u_{2}$ edge, the value of $a_{21} ; a_{22} ; a_{25}$ and $a_{26}$ coefficients belonging to $x_{1} ; x_{2} ; x_{5}$ and $x_{6}$ variable is 1 . The value of the other coefficients in the given inequality is 0 . Applying this consideration, only the corresponding part-flows have been considered in case of each network component. In accordance with the introduced considerations, $\underline{A}$ matrix have been defined as follows:

$$
\stackrel{A}{=}=\left[\begin{array}{llllllll}
0 & 0 & 0 & 0 & 0 & 0 & 0 & 0 \\
1 & 1 & 0 & 0 & 1 & 1 & 0 & 0 \\
1 & 1 & 1 & 1 & 0 & 0 & 0 & 0 \\
1 & 0 & 1 & 0 & 1 & 0 & 0 & 1 \\
0 & 0 & 0 & 0 & 0 & 0 & 0 & 0 \\
0 & 1 & 0 & 1 & 1 & 0 & 0 & 1 \\
0 & 1 & 1 & 0 & 0 & 1 & 0 & 1 \\
0 & 0 & 0 & 0 & 0 & 0 & 0 & 0 \\
0 & 1 & 0 & 1 & 0 & 1 & 1 & 0 \\
0 & 0 & 1 & 1 & 0 & 0 & 1 & 1 \\
0 & 0 & 0 & 0 & 1 & 1 & 1 & 1 \\
0 & 0 & 0 & 0 & 0 & 0 & 0 & 0
\end{array}\right]
$$

Finally, the objective function have been defined. The aim was to minimize the total travel time of the vehicles go through the network in an investigated sample time period, so the objective function have been defined as the sum of products of total traffic and constant travel time characterizing the edges of the graph, as indicated in Eq. (7).

$$
F=\sum_{j=1}^{12} X_{u_{j}} * t_{j} \rightarrow \text { min. }
$$

Equations (1)-(7) describe the optimization process considering the constraining and boundary conditions as well as the objective function with linear formulas, based on static conditions and assumptions.

\section{Results}

The example has been solved based on the introduced method with the above mentioned assumptions to verify the applied method. Static parameters of the example have been considered as follows. In Table 1, transport demands between the OD zones have been introduced.

Table 1 Matrix of transport demands of the system

\begin{tabular}{ccc}
\hline & W & $\mathrm{Z}$ \\
\hline $\mathrm{S}$ & 10 & 5 \\
$\mathrm{~T}$ & 15 & 20 \\
\hline
\end{tabular}

Values of capacity and travel time constants of graph edges of the investigated example have been defined in Table 2.

Table 2 Data of the edges of the investigated network

\begin{tabular}{ccc}
\hline Edges $\left(u_{j}\right)$ & Capacity $\left(c_{j}\right)$ & Travel time $\left(t_{j}\right)$ \\
\hline$u_{1}$ & 20 & 3 \\
$u_{2}$ & 25 & 3 \\
$u_{3}$ & 25 & 3 \\
$u_{4}$ & 25 & 3 \\
$u_{5}$ & 30 & 3 \\
$u_{6}$ & 25 & 3 \\
$u_{7}$ & 20 & 3 \\
$u_{8}$ & 25 & 3 \\
$u_{9}$ & 25 & 3 \\
$u_{10}$ & 25 & 3 \\
$u_{11}$ & 35 & 3 \\
$u_{12}$ & 25 & 3 \\
\hline
\end{tabular}

MATLAB software has been used for the optimization. Based on the intlinprog command of the software it was possible to constrain the value of the variables to integers, and define the further necessary constraining conditions. With the help of the software, the optimized values of the variables and the objective function resulted as follows: 


$$
\begin{aligned}
& x_{1}=10 ; x_{4}=5 ; x_{5}=15 ; x_{7}=20 \\
& x_{2}=x_{3}=x_{6}=x_{8}=0 ; F=510
\end{aligned}
$$

\section{Conclusions}

Static system optimum of linear traffic distribution problem has been introduced in the article, assuming an intelligent and autonomous transportation system, where the trip distribution tasks and route choice decision processes are under the control of the system. Linear programming framework of the problem has been elaborated. Model variables were the traffic volumes of alternative routes connecting OD zones, the minimized objective function has been derived from the sum of products of part-flows and related travel times, considering all constraining factors.

The description of the applied method and its verification have been presented in a simplified model example. In the result section it has been shown, that in case of the introduced static conditions the approach is applicable to determine the optimal solution of the traffic distribution problem in a given sample time period of an autonomous transportation system. Static system optimum has been reached as required transport demands between the zones are to be satisfied as follows: all demands between zones $\mathrm{S}$ and $\mathrm{W}$ have to be loaded on $U_{S W 1}$; between $\mathrm{S}$ and $\mathrm{Z}$ on $U_{S Z 2}$; between $\mathrm{T}$ and $\mathrm{W}$ on $U_{T W 1}$; and between $\mathrm{T}$ and $\mathrm{Z}$ on $U_{T Z 1}$. In this case, all demands are satisfied beside the minimum workload of the system $(F=510)$ that is derived from traffic volumes and travel time of the edges of the network. Sankey diagram of the results have been presented in Fig. 2, to illustrate the optimal solution.

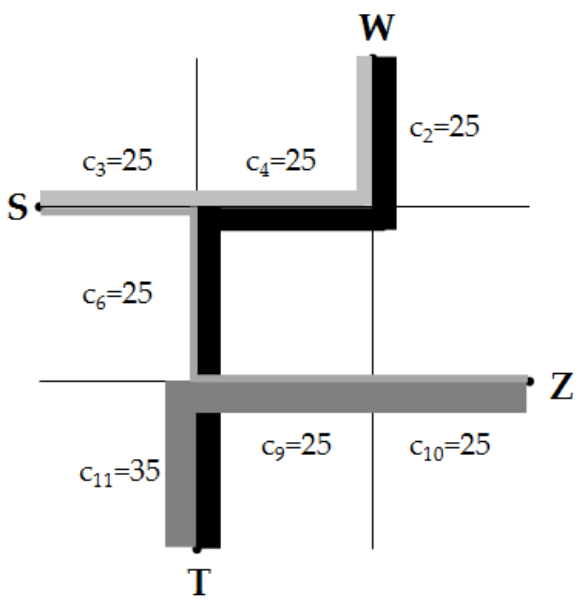

Fig. 2 Sankey diagram of the results representing the static system optimum

Authors' future work will focus on the extended implementation of the method on more complex transportation networks, to prove the applicability of linear programming problems on transport related examples. Furthermore, we aim to examine further application possibilities to evaluate routes of networks from the aspect of road safety and/or road toll structure by changing the model variables.

\section{References}

Díaz-Madroñero, M., Mula, J., Peidro, D. (2017). A mathematical programming model for integrating production and procurement transport decisions. Applied Mathematical Modelling. 52, pp. 527-543. https://doi.org/10.1016/j.apm.2017.08.009

Harasimowicz, S., Janus, J., Bacior, S., Gniadek, J. (2017). Shape and size of parcels and transport costs as a mixed integer programming problem in optimization of land consolidation. Computers and Electronics in Agriculture. 140, pp. 113-122.

https://doi.org/10.1016/j.compag.2017.05.035

Kumar, P., Rosenberger, J. M., Iqbal, G. M. D. (2016). Mixed integer linear programming approaches for land use planning that limit urban sprawl. Computers \& Industrial Engineering. 102, pp. 33-43. https://doi.org/10.1016/j.cie.2016.10.007

Ma, J., Li, X., Zhou, F., Hao, W. (2017). Designing optimal autonomous vehicle sharing and reservation systems: A linear programming approach. Transportation Research Part C: Emerging Technologies. 84, pp. 124-141. https://doi.org/10.1016/j.trc.2017.08.022

Yakimov, M. (2017). Optimal Models used to Provide Urban Transport Systems Efficiency and Safety. Transportation Research Procedia. 20,pp. 702-708. https://doi.org/10.1016/j.trpro.2017.01.114

Yang, X. S. (2016). Engineering Mathematics with Examples and Applications. Academic Press. ISBN: 978-0-12-809730-4 